\title{
A simple derivation of the Gompertz law for human mortality.
}

\author{
B. I. Shklovskii \\ William I. Fine Theoretical Physics Institute, University of Minnesota, Minneapolis, Minnesota 55455
}

(Dated: October 26, 2018)

\begin{abstract}
The Gompertz law for the human mortality rate as a function of age is derived from a simple model of death as a result of an exponentially rare escape of abnormal cells from the immunological response.
\end{abstract}

Human life is finite as are the lives of some well known physical objects for example radioactive nuclei. The probability, $S(t)$, that a given nucleus will survive time $t$ is

$$
S(t)=\exp \left(-t / t_{0}\right)
$$

If survival of humans were governed by the same law with, for example, $t_{0}=70$ years we would have millions of people with the age, say, $5 t_{0}=350$ years. However, the longest recorded human life lasted only 122 years. Apparently, the law of human mortality should be drastically different from Eq. (1).

The famous astronomer Halley $\underline{\underline{1}}$ and the great mathematician Euler ${ }^{2}$ were the first to attack this problem, but the law was empirically found only in 1825 by the actuary and self-taught mathematician Benjamin Gompertz ${ }^{3}$. If $S(t)$ is probability of a human surviving till age $t$ years then the mortality function $\mu(t)$ is defined as

$$
\mu(t)=-d \ln S(t) / d t
$$

Gompertz found that when childhood diseases are overcome $(t>25)$ the statistics of mortality obeys the law

$$
\mu(t)=\mu(25) \exp \left([t-25] / t_{1}\right),
$$

where $t_{1} \simeq 10$. This law should be contrasted with the constant mortality function $\mu(t)=1 / t_{0}$ following from Eq. (11). The Gompertz law means that $S(t)$ decays double exponentially, much faster than Eq. (11), practically eliminating people older than 122 in the world population. Statistical data for many countries and three centuries confirm the Gompertz law in the range of $\mu(t)$ covering more than three orders of magnitude (see Refs. ${ }^{4}$ and references therein). The law works also for different species such as rats, mice and fruit flies. Thus, the Gompertz law emerges as one of the greatest quantitative laws of biology.

There were a number of attempts to derive the Gompertz law. The most quoted derivation uses the langauge of the reliability theory designed for man-made machines ${ }^{\underline{5}}$ and is quite complicated. Here, we would like to suggest a simple version of derivation of Eq. (3), based on a naive understanding of immunology. Thinking about cells, we assume that a population of defective cells (mutated cells that do not fulfill their normal function, cancer cells undergoing uncontrolled proliferation, cells that produce masses of defective protein forming amyloids and senile plaques in aged tissues leading to diseases like Alzheimer) becomes fatal if the normal organism defense system gives this population a time $\tau$ necessary to reach a critical size. Let us assume that at age 25 , bad cells would encounter strong and fatal immune response during time $\tau$ on average $N_{0} \gg 1$ times. (We can imagine a bad cell as criminal who is being eliminated at any encounter with randomly patrolling policemen (macrophages, natural killers, or apoptosis), before growing strong enough to successfully resist them. Until this time, on average, he would meet $N_{0}$ policemen.) For absolutely random encounters, the probability $P(N)$ of the number $N$ of encounters with immune response system during time $\tau$ is given by the Poisson distribution,

$$
P(N)=\frac{N_{0}^{N} \exp \left(-N_{0}\right)}{N !}
$$

where $N_{0}$ is the average value of $N$. The probability of a population of defective cells reaching the critical size and causing the death of the host at age 25 is $\mu(25)=P(0)=\exp \left(-N_{0}\right)$. It is known that $\mu(25)$ is of the order of $310^{-4}$. This means that, indeed, $N_{0} \simeq 8 \gg 1$. Let us assume now that the immune response slowly weakens with the age so that at $t>25$ the average number of encounters during "microscopic" time $\tau$ decreases linearly as $N_{0}(t)=N_{0}-(t-25) / t_{1}$. This may happen due to accumulation of mutations in immune response cells, their limited potential for self-renewal, or an overall decay of the organism energetics. (In the language of criminals and policemen, this would mean slow decay of the number of patrolling policemen, for example, due to budget restrictions). Substituting this $N_{0}(t)$ instead of $N_{0}$ into the Poisson formula for $P(0)$ we arrive at $\mu(t)=\exp \left[-N_{0}(t)\right]$ and, therefore, at Eq. (3).

Notice that a relatively small change of $N_{0}(t)$ leads to a very strong exponential growth of $\mu(t)$. A linear decay of $N_{0}(t)$ can be the initial stage of an exponential relaxation $N_{0}(t)=N_{0} \exp \left(-[t-25] / N_{0} t_{1}\right)$. In this case, at large ages $t \sim 100$ we arrive at downward deviations of $\mu(t)$ from the Gompertz law. This qualitatively agrees with somewhat slower than Eq. (3) growth of mortality at $t>100$ cited in $4.5,6.7$. These empirical deviations from Eq. (3) are still under discussion because of relatively poor statistics for $t>100$.

I am grateful to M. Azbel, A. Finkelstein, A. Chklovski, D. Chklovskii, S. Grigoryev, I. Ruzin, L. Shklovskii and D. Stauffer for useful discussions. 
1 E. Halley, Phil. Trans. Roy. Soc. 17, 596 (1693).

${ }^{2}$ L. Euler, Histoire de l'Academie Royale des Sciences et Belle - Letters, 144 (1760).

3 B. Gompertz, Philos. Trans. Roy. Soc. Lond. A 115, 513 (1825).

4 D. Stauffer, The Complexity of Biological Ageing. p. 131 in: Thinking in Patterns, ed. by M.M. Novak, World Scientific,
Singapore 2004; cond-mat/0310038

${ }^{5}$ L. A. Gavrilov, N. S Gavrilova, J. Theor. Biology 213, 527 (2001).

${ }^{6}$ M. Azbel, Physica A 249, 472 (1998).

7 R. M. C. de Almeida, S. Moss de Oliveira, and T. J. P. Penna, Physica A 253, 366 (1998). 